\title{
Blind man draws using diminution in three dimensions
}

\author{
JOHN M. KENNEDY and IGOR JURICEVIC \\ University of Toronto, Toronto, Ontario, Canada
}

\begin{abstract}
Can the principle of convergence in three spatial dimensions be reflected in drawings by the congenitally blind? A man who had been totally blind since birth was asked to draw scenes such as a tabletop with three cubes receding to the observer's left side. He used converging lines to show the tops of the cubes receding in depth. He drew the cubes to the left smaller than the cube in front of the observer. He drew faces of cubes to the left with tilted lines, pointing to below the front face of the cube in front. The result approximates three-point perspective. We note that the directions of objects from a vantage point in touch converge much as they do in vision.
\end{abstract}

Here, we report that a man who is congenitally totally blind used pictorial convergence to show depth and drew a scene with three cubes in a manner approximating threepoint perspective. This use of convergence in a picture of a cube is of general interest, since the question of what can imply depth to blind and sighted observers has been debated since William Molyneux in the 17th century posed the theoretical question to John Locke (Lopes, 2003, pp. 178-180). We argue that the directions of objects from the observer's vantage point are relevant to pictorial convergence for the sighted and the blind.

Pictures can be made in a raised form for use by the blind (Axel \& Levent, 2003). People totally blind from birth who have had little or no experience drawing make outline pictures of objects after a few minutes practice with a raised-line drawing kit (Kennedy, 1993; Heller, 2000; Millar, 1994). Two blind people who had extensive practice drawing have been tested. One (a blind child in Rome) draws objects using parallel projection (Kennedy, 2003); that is, nearby and more distant spaces of the same size in the scene require equal-sized spaces in the picture. One (an adult in New York) uses convergent lines in accord with one-point perspective (Kennedy \& Juricevic, 2003); that is, parallels in one dimension, such as a flat ground, converge to one point. (For the sighted, a familiar picture in one-point perspective would be a depiction of railroad tracks, the lines for the rails converging toward the line for the horizon.)

The use of convergent lines in a picture to represent parallels in a depicted scene occurs late in the develop-

Joan Eroncel must be singled out for recognizing that E.A. should be given scholarly attention. We thank E.A. for participating in this study. We offer this paper to Rudolf Arnheim, in his 100th year. Correspondence concerning this article should be addressed J. M. Kennedy, University of Toronto, 1265 Military Trail, Toronto, ON, M1C 1A4 Canada (e-mail: kennedy@utsc.utoronto.ca). ment of drawing skills in the sighted child-typically, after the age 9 or 10 years - and becomes common only in early adolescence (Willats, 1997). The amount of convergence is highly imprecise unless a calculation method has been formally taught. Here, we show that a blind man used one-point convergence in this imprecise fashion in several pictures. He then sketched a house in rough twopoint perspective. That is, parallels in two planes (the front and end walls of the house) converged in two directions, one to the left and one to the right of the center of the picture. He went on to use convergence, size diminution, and tilted lines in dealing with depth in the three dimensions of space - $x$ (horizontal), $y$ (vertical), and $z$ (depth) - in a picture of cubes. We suggest that convergence, diminution, and tilt are related devices that fit with the directions of parts of a scene with respect to an observer, albeit one without sight. We speculate that our informant generalized his remarkably fluid use of one-point convergence to solve the specific tasks that we asked him to undertake.

Consider a cube on a table, sitting directly in front of the observer but below his or her vantage point. Let the cube move to the observer's left. Now let it move further to the left. To show this set of cubes, such devices as omitting or showing side faces are needed to reveal which cube is to the fore and which are increasingly distant to the left. Tops, fronts, and sides of cubes need to be drawn systematically. This task is challenging to sighted observers. It can be solved using devices having to do with convergence in the $x, y$, and $z$ dimensions. That is, the convergence occurs not only at points to the left and right of the picture's center, but also at a point below the center of the picture. The three cubes will be called the cubes to the front, to the left of the one in front, and leftmost in our analysis.

This task was given to a blind man who has made pictures frequently ever since he was a child. (His parents gave him drawing materials to stop him from scribbling on the furniture, he reports.) First, he was given a series of drawing tests, several pertinent to one- or two-point 
convergence. Then the three-cubes test was given. The question was whether he would use convergence to show distance in several directions.

\section{METHOD}

\section{Subject}

E.A., 51 years of age, is a congenitally totally blind man from Turkey. He has an incomplete grade school education. One eye is missing, and the other is a microball that has no light sensitivity. Medical opinion is that he has never had light sensitivity. He had previously been asked to draw moving wheels (Kennedy \& Merkas, 2000). As a child, he drew on his parents' furniture, in dirt or sand (Motluk, 2005), and on paper, making thick indented lines. He reports having asked people about pictures, and he recalls that in his teens or early twenties, he was told the parallel sides of roads can be drawn as converging. His drawings are not rote copies, since he can explore a novel object and produce an excellent, easily recognized picture of it.

The present authors have seen over 50 pictures made by E.A., at his own behest. Joan Eroncel, who translated instructions into E.A.'s native Turkish for this study, has seen many hundreds. In none is two- or three-point perspective evident. However, he drew distant objects, such as houses, smaller than ones in the foreground. E.A. drew receding sides of houses as converging (Motluk, 2005) - that is, in one-point perspective-while drawing the fronts of the houses with parallel lines.

\section{Procedure}

E.A. was asked to undertake 15 drawing tasks, including the following: one pencil crossing on top of another; people standing, walking, and lying down; an array of three objects from different vantage points; cars at different distances on a road; receding rows of glasses; a table and chairs; a house; and three cubes, one of which was placed in front of him (an 8.5-cm-wide wooden cube set on the table at which he was seated), one described as being to the left (again demonstrated with the wooden cube, moved double the cube's width to the left), and one described as being further to the left (again demonstrated, with the cube moved another two widths to the left). Here, the results for the picture tasks relevant to one- and twopoint convergence will be noted, prior to presenting the three-cubes task. E.A. was not subjected to any time pressure. Times to complete the drawings ranged from about half a minute to several minutes. Time to start a drawing after the task had been given ranged from a delay of a few seconds to about $2 \mathrm{~min}$ (for the three-cubes task).

\section{RESULTS}

As is shown in Figure 1, E.A. drew the car in the distance small, showed the fronts of each car as being smaller than the rears, and used lines for the roadsides that converged with distance. Also, he drew two rows of glasses (with three glasses per row) converging, as had Tracy, a blind adult from New York (Kennedy, 2003). The further glasses were drawn smaller, closer together, and higher up the page. He also drew the sides of a table converging. In sum, in Figure 1, one-point perspective is present in the three tasks.

Figure 2 shows drawings of a model house. Top left, the house is shown from the front. The rectangular roof is drawn as converging. Top right is the house from the end wall, showing its peaked roof. On the bottom left, the house is shown from above, with the roof drawn as adjoining rectangles, in parallel projection. Bottom right are two drawings of the house situated with a corner in front of the observer. E.A.'s first drawing is the upper one, with parallel oblique lines showing the sides as receding in depth. The second is a sketch he made while explaining that each of the sides meeting at the corner can be drawn as "narrowing." It is in two-point perspective, the first ever such drawing observed from E.A.

Figure 3 shows E.A.'s three-cubes drawing. The cube on the right is the one in front of the observer. The top converges upward on the page. The top of the middle cube (left of the cube in front) is drawn as converging up the page and to the right. It converges to the same area as do the lines for the top of the cube in front. The top of the cube on the left is drawn with highly tilted lines, converging sharply to the area above the cube in front.

The vertical face of the cube in front is drawn as converging downward slightly. (This is a hallmark of a tilted picture plane.) The angle between its left and right sidelines (measured between straight lines made by joining the
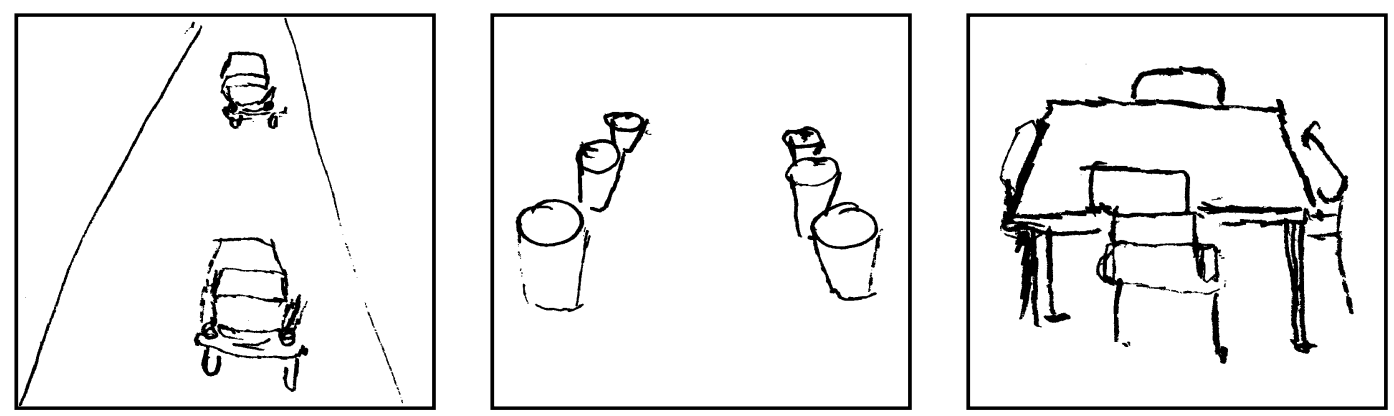

Figure 1. Drawings by E.A. using convergence in the $z$ dimension: a road with two cars, rows of glasses, and a table and chairs. 


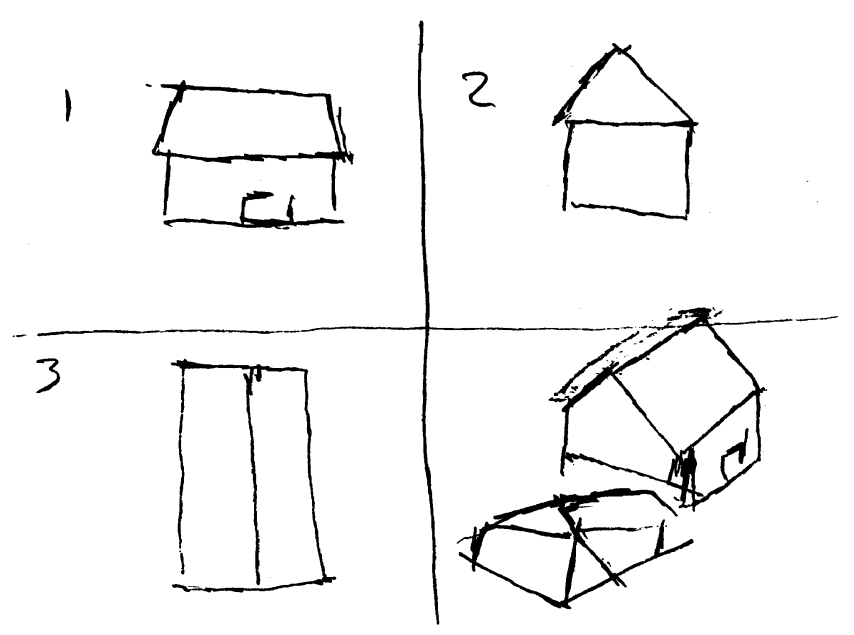

Figure 2. Drawings of a house by E.A. using parallel projection, convergence in one direction, and convergence in two directions.

line junctions at the top and bottom of the sidelines) is $7^{\circ}$. This may be E.A.'s second use of two-point perspective, but the convergence is slight. For present purposes, we emphasize that lines for three vertical edges of the cube to the left are tilted $-15^{\circ}-20^{\circ}$ to the vertical, bottom toward the right, in a five o'clock position, pointing to the area below the cube in front. The three vertical edges of the leftmost cube are tilted in a similar way (each about $20^{\circ}$ to the vertical). As a result, if the lines for the front face of the cube to the front are extended downward and the tilted lines for the verticals of the cubes to the left are extended, they converge.

The sizes of the cubes diminish as they recede to the left. For convenience, take the line for the left edge of the cube to the front as a standard. The line for the same edge of the cube to the left is .62 of the standard. The same edge in the leftmost cube is .4 of the standard. As a result of this diminution, an imaginary line connecting the tops of the three cubes and another imaginary line connecting the three bottoms converge to the left. In addition, the set of cubes is flanked top and bottom by lines converging to the left, suggesting the support surface receding to the left. These were drawn last.

The lines for the three tops converge to a region above the cube in front, the sidelines of the vertical faces point to the region below, and sizes diminish to the left. The tops converge to show $z$, the verticals on the left tilt in showing $y$, and sizes diminish (converge) for $x$, taking in all three spatial dimensions.

\section{DISCUSSION}

E.A.'s drawings include one- and two-point perspective. In the three-cubes drawing, he shows the distances between lines as diminishing in the three dimensions of space in several ways, approximating three-point perspective.

It may be especially revealing that the cube verticals were shown as tilting and, thereby, converging. This suggests a tilted picture plane (Kubovy, 1986), since a set of verticals project as parallel lines if the picture plane is parallel to them. The supposition that a tilted picture plane is relevant was reinforced by E.A.'s behavior while drawing the three cubes. He held his hands, tilted, halfway between his face and the table - something he did not do for any other drawing. He posed for about a minute, evidently considering the task. He then began drawing steadily and decisively. We suggest that he was considering the directions of parts of the scene from the vantage point of his head and how these would intersect the plane defined by his hands.

E.A. said that the tasks with the glasses, the table and chairs, the house from different vantage points, and the three cubes were novel. If so, Figures 2 and 3 may, indeed, be his first use of two-point and an inventive approximation to three-point perspective. He previously had drawn receding sides of an object as converging, but to our knowledge, always as if one side of an object was parallel to the picture plane and one was receding. In contrast, a house with its corner forward has its two orthogonal sides both receding from the picture plane. What is new is that E.A. applied the convergence principle to both sides, generalizing aptly from the one-point case, and then to three sides of a cube. He generalized his use of convergence to novel tasks. He was not restricted to examples learned by rote, if the house corner and three-cubes tasks were novel. If he generalized from comments about drawing the sides of roads as converging with distance - a modest level of supportive feedback and instruction - integrating this with tactile information about direction, his achievement is noteworthy.

E.A. considers that cubes to the left of straight-ahead can be drawn smaller the further away they are. However, if the $x$ dimension of the picture plane was parallel to the front faces of the cubes, all their fronts would all be drawn the

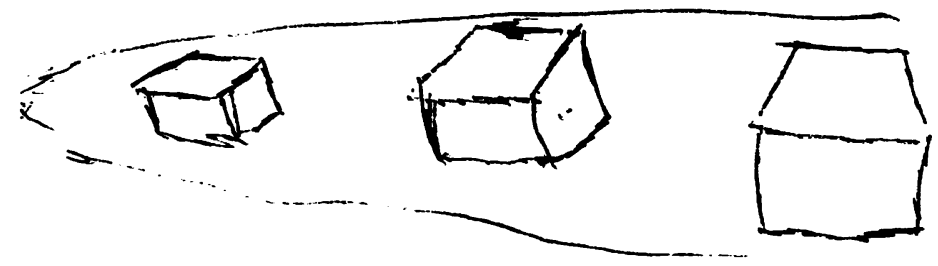

Figure 3. Drawing of three cubes, using devices to show recession in three dimensions. One cube is depicted as straight ahead (right), one is to its left (middle), and one is further to its left. 
same size. To depict objects to the left as smaller, the picture plane should be rotated out of the frontal plane, counterclockwise viewed from above. However, the frontal cube is drawn with parallel horizontal lines, as if the picture plane is not rotated. E.A. may simply have argued that since the cubes to the left are receding in distance, they should converge in size, much like his cars in Figure 1.

Why was diminution with distance appreciated by E.A.? A major reason could be that convergence is a matter of direction. That is, just as visible objects lie in different directions around a seated sighted person, they lie in different directions for a blind observer reaching out to touch them. Visual and tactile reaching directions are identical. Both sighted and blind observers use direction in daily routines. For both kinds of observers, as an object recedes, the directions to its sides come together. What is important is that the spatial skill underlying the routines may support use of an approximation to three-point perspective in the blind, as well as in the sighted.

Although E.A.'s use of kinds of pictorial diminution may be rooted in common, everyday skills with direction, we speculate that it is nevertheless notable for theories of development. Direction information is used in connection with convergence in drawings only after very considerable development of drawing skills in the sighted child (Willats, 1997), which normally reaches a plateau at oneor two-point perspective in the sighted. In many years of testing thousands of sighted children and adults drawing cubes, we have not seen an instance of a sighted person who draws in three-point perspective without having been explicitly taught.

E.A. did not calculate exact rates of diminution and exact directions of convergence. But the three-cubes drawing shows the space between lines as diminishing roughly in the right directions to conform to the three dimensions of space.

That E.A. is so advanced in drawing development suggests that blind children and adults should be given pictorial opportunities in plenty. Blind and sighted children may be on the same drawing development ladder, likely to go through the same stages.

E.A. selects his own topics and draws at his own behest, depicting novel objects successfully in the judgment of the sighted. He indicates that he receives modest feedback from observers in the form of interest and praise, and we and Ms. Eroncel have observed this in several circumstances. To produce pictorial forms, he does not depend on being taught specific drawings, although he notes that if he wants to know the color of an object, he has to ask. We hypothesize that his skills with forms, suitable vantage points of the observer, and kinds of projection are largely of his own devising, in reliance on direct interaction with objects and lots of practice in drawing. However, the role of feedback, of tasks and solutions from other people, and of such matters as the availability of models for the blind and the sighted deserves a great deal of attention, and our hypothesis should be recognized to be a conjecture.
We should note that the house corner task is especially pertinent to a key principle. E.A. applied the convergence evident in a one-point perspective drawing of the roof to the front and sides, in two-point perspective. Just so, in the Renaissance, exact perspective is thought to have been discovered by the architect Brunelleschi when drawing a building in Florence (the Baptistry) that had two receding orthogonal sides (Kubovy, 1986). The selection of a house corner task favors development, it might be argued. Once E.A. generalized convergence from one to two dimensions, to cope with the house corner, the way to three dimensions for the cubes may have been facilitated. If so, the possible role of a facilitating task needs to be made clear. In addition, since we have given this task to other blind people (Kennedy, 2003; Kennedy \& Juricevic, 2003) but they did not invent two-point convergence, it seems likely that there is just as much of a role in development for a prepared mind, highly practiced with principles used in certain tasks, as for new tasks relevant to a key principle. That is, E.A.'s regular use of one-point convergence in several tasks may reveal that he was ready to advance developmentally, given a welldefined and suitable task to which a variation of one-point convergence could be applied.

In sum, in his drawings, E.A. used diminishing distances between lines to depict recession in one, two, and three dimensions. Convergence in direction due to increasing distance from a vantage point matters to the blind and the sighted. Pictorial diminution in all three spatial dimensions is an advanced development, which we suggest E.A. reached more on his own initiative than through instruction. The developmental route he has taken may be open to the blind for the same reason as it is to the sighted. Directions of objects from a vantage point matter to both touch and vision.

\section{REFERENCES}

Axel, E., \& Levent, N. (2003). Art beyond sight. New York: AEB and AFB Press.

Heller, M. A. (ED.) (2000). Touch, representation and blindness. Oxford: Oxford University Press.

Kennedy, J. M. (1993). Drawing and the blind. New Haven, CT: Yale University Press.

Kennedy, J. M. (2003). Drawings by Gaia, a blind girl. Perception, 32, 321-340.

Kennedy, J. M., \& Juricevic, I. (2003). Haptics and projection: Drawings by Tracy, a blind adult. Perception, 32, 1059-1071.

Kennedy, J. M., \& Merkas, C. E. (2000). Depictions of motion devised by a blind person. Psychonomic Bulletin \& Review, 7, 700-706.

Kubovy, M. (1986). The psychology of perspective and Renaissance art. Cambridge: Cambridge University Press.

Lopes, D. M. M. (2003). Are pictures visual? A brief history of an idea. In E. Axel \& N. Levent (Eds.), Art beyond sight (pp. 176-185). New York: AEB and AFB Press.

Motluk, A. (2005). Seeing without sight. New Scientist, 185, 37-39.

Millar, S. (1994). Understanding and representing space. Oxford: Oxford University Press.

Willats, J. (1997). Art and representation. Princeton, NJ: Princeton University Press.

(Manuscript received February 21, 2005; revision accepted for publication October 17, 2005.) 